\title{
Reply by the authors to the discussion by Nakatsuka
}

\author{
Jun Matsuo ${ }^{1,4}$ Nobuhiro Isezaki $i^{2,3,4}$ \\ ${ }^{1}$ OYO International Corporation Yushima 1-Chome Bldg. 4F, 6-3 Yushima 1-Chome, Bunkyo-ku, Tokyo 113-0034, Japan. \\ ${ }^{2}$ Chiba University, 1-33 Yayoi-cho, Inage-ku, Chiba 263-8522, Japan. \\ ${ }^{3}$ Tokai University, 3-20-1 Orido Shimizu-ku, Shizuoka 424-8610, Japan. \\ ${ }^{4}$ Corresponding authors. Email: matsuo@oyointer.com; nisezaki@earth.s.chiba-u.ac.jp; isezaki@tokai-u.jp
}

We appreciate the questions, and also SEGJ who provides this opportunity to explain our study conception. We hope this discussion deepens mutual understanding.

\section{Discussion - 1}

The questioner pointed out, 'Their figures 3 and 4 are correct although the description of the horizontal axis of figure 4 could be clearer. It is obvious that the relative error $\varepsilon_{\mathrm{T}} / T I A(=(T I A-P T A) /$ $T I A$ ) becomes infinite where $T I A=0$. However, the situation is not as severe as their assertion implies, because such a large error occurs only in a quite limited region near $P T A=0$, but the result of analyses of magnetic anomalies is commonly more dependent on the locations and values of the high and low anomaly peaks, where the approximation above is fully valid. To illustrate this, I show a simple synthetic model.'

What we point first in this paper is that TIA cannot be used for the physical analyses because it is not a vector but just a scalar.
Figure 4 in our paper showed the relationship between TIA and $\varepsilon_{\mathrm{T}}$ for the $2 \mathrm{D}$ case. Considering the actual $3 \mathrm{D}$ case, it is almost impossible to evaluate $\boldsymbol{\varepsilon}_{\mathrm{T}}$ by the method of model analysis because there are the infinite complex combinations of the shape, direction, and intensity of magnetization.

Evaluation of $\varepsilon_{\mathrm{T}}$ can be done only using the observed TA (three component anomalies). Figure F-1 shows the result of evaluation of $\boldsymbol{\varepsilon}_{\mathrm{T}} / \mathrm{PTA}$ (TIA) using the observed TA at Aogashima Island. It is clear that at the $\beta$ around $1^{\circ}, \boldsymbol{\varepsilon}_{\mathrm{T}} / \mathrm{PTA}(\mathrm{TIA})$ reaches more than $10 \%$ and sometimes more than $100 \%$. $\varepsilon_{\mathrm{T}} / \mathrm{PTA}$ (TIA) directly connects to $\mathrm{dM} / \mathrm{M}$, the relative error for magnetization $\mathrm{M}$ as mentioned later.

\section{Discussion - 2}

The questioner showed Figure D-1, and he showed simple dipole model analysis. In addition, his conclusion revealed that

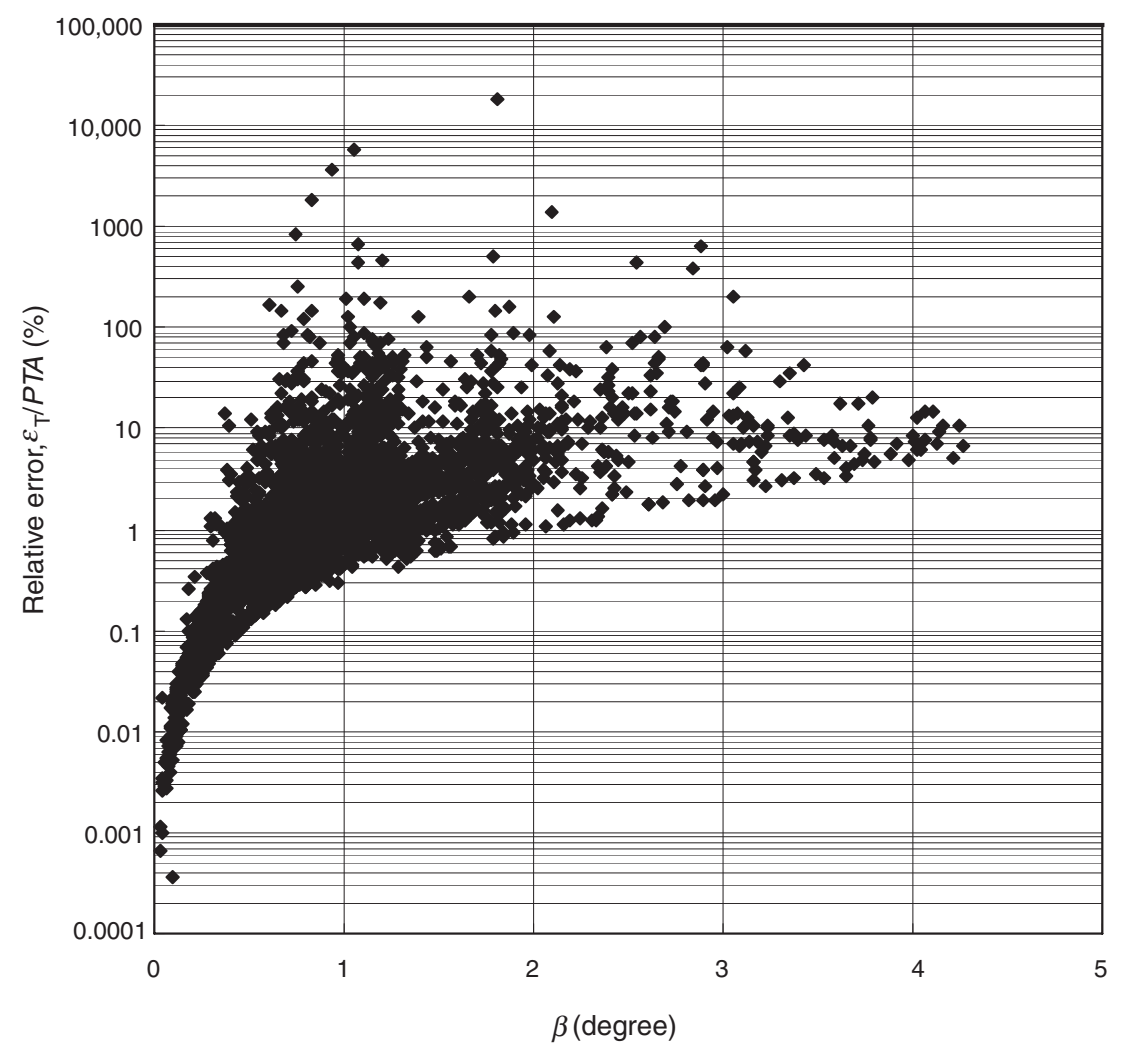

Fig. F-1. The actual result of relationship between $\beta$ and relative error, $\varepsilon_{\mathrm{T}} / P T A$. (At altitude $550 \mathrm{~m}$ ). This figure shows the relationship between $\beta$ and the relative error, $\varepsilon_{\mathrm{T}} / P T A$, of the magnetic field in actual data of Aogashima. For example, when $\beta$ is around one degree, the relative error is from $0.5 \%$ to $100 \%$. This figure shows that the data gather densely at $0.5^{\circ}<\beta<1.5^{\circ}$ and as $\beta$ increases ( $|\mathbf{T A}|$ increases), the relative error, $\varepsilon_{\mathrm{T}} / P T A$, increases up to $10 \%$. 
the peak value of $\varepsilon_{\mathrm{T}}$ is $\sim 14 \mathrm{nT}$, although the same difference may be caused by the position inaccuracy of $6 \mathrm{~m}$ in horizontal.

He supposes the difference, $\varepsilon_{\mathrm{T}}=\mathrm{TIA}-\mathrm{PTA}$, is small. We must note that this example of model calculation is too simple to simulate the actual situation of magnetization. One point magnetic moment is very far from the representative of actual complicated 3D structure. Figure 4 in our paper shows the relationship between TIA and $\varepsilon_{\mathrm{T}}$ just for the $2 \mathrm{D}$ case. We can easily guess the relationship with $\mathrm{st}$ and $\beta$ is more complex in the actual 3D case.

Evaluation of $\boldsymbol{\varepsilon}_{\mathrm{T}}$ can be done only using the observed TA.

\section{Discussion - 3}

The questioner showed the inversion result in Table D-1 (see Table D-1 in Discussion paper, page 122). He showed the error level of magnetic moment is $0.15 \%$ in magnitude and $0.34^{\circ}$ in direction based on the assumption that PTA $=$ TIA.

The relative error strongly influences to the magnetization analysis error. The simple explanation is as follows; $\mathbf{M}$ is obtained from TA or PTA using the physical formulae, $\mathbf{T A}=\operatorname{ct}(r) * \mathbf{M}$, or PTA $=\operatorname{cp}(r) * \mathbf{M}$ where $\operatorname{ct}(r), \operatorname{cp}(r)$ are the function of $r$, the distance between $\mathbf{M}$ and the observation point and the shape of $\mathbf{M}$. Under the assumption that $P T A=T I A, \mathbf{M}^{\prime}$ is obtained from

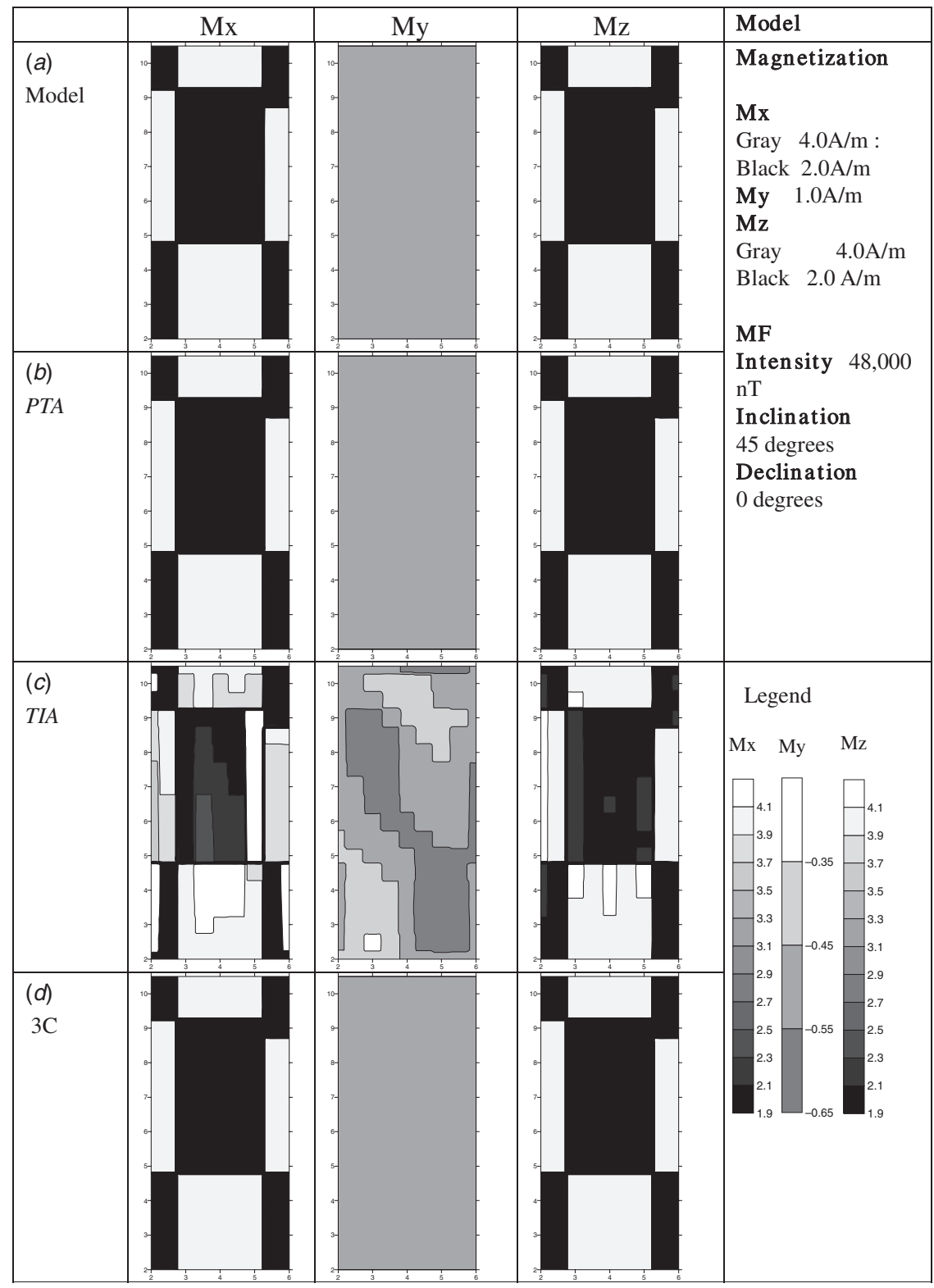

Fig. F-2. Figure 5 (Isezaki and Matsuo, 2009) with revised legend as written - Figure 5: Inversion results for three components of magnetization, using PTA, TIA and three-component anomalies, for a simple block model. (a) Model; (b) Inversion result using PTA as the observed data, (c) Inversion result using TIA as the observed data; $(d)$ Inversion result using three-component anomalies as the observed data. The magnetization model was a flat plate made up of an aggregation by blocks. The thickness of the plate was $2000 \mathrm{~m}$; the length and width of each block was $500 \mathrm{~m}$. The total number of prismatic blocks was 162 (18 north $\times 9$ east), and as each block had three components of magnetization, then there were 486 unknowns. Magnetic anomaly data on planes 200,350,450,500, and $550 \mathrm{~m}$ above the surface of the plate were used. The total number of observed (calculated) data was 6,377. To calculate TIA and PTA, the following parameters were assumed. (1) $M F=48,000 \mathrm{nT}$; (2) The declination was $0^{\circ}$; (3) The inclination was $45^{\circ}$. 
$T I A=\operatorname{cp}(r) * \mathbf{M}^{\prime}$. However $\mathbf{M}^{\prime} \neq \mathbf{M}$ because $P T A \neq T I A$. Then the relative error occurs as follows,

$$
\begin{aligned}
\text { relative error } & =(T I A-P T A) / P T A=\mathrm{cp}(r) \\
& *\left(\mathbf{M}^{\prime}-\mathbf{M}\right) / \mathrm{cp}(r) * \mathbf{M}=\mathrm{d} \mathbf{M} / \mathbf{M} .
\end{aligned}
$$

Figure F-1 shows that the relative error evaluated from measured TA reaches more than $10 \%$, sometimes more than $100 \%$ around $\beta=1^{\circ}$.

In the text, the definition of relative error is $\varepsilon_{\mathrm{T}} / T I A$, however, the definition $\varepsilon_{\mathrm{T}} / P T A$ for the relative error gives almost the same behaviour as $\varepsilon_{\mathrm{T}} /$ TIA for the much smaller $\beta$.

\section{Discussion - 4}

The questioner pointed out, 'The validity of approximating observed TIA anomalies with PTA anomalies is mentioned in most textbooks (e.g., Blakely, 1995, p. 179; SEGJ, 1999, p. 483). As this approximation is too common among exploration geophysicists concerned with mobile magnetic surveys, the description of the effect of approximation is usually omitted.'

Blakely (1995), Bhattacharyya (1964), Talwani (1965) and so on assumed in their papers that $|\mathbf{T F}| \gg|\mathbf{T A}|$, and $T I A=P T A$, although they did not know how large $|\mathbf{T A}|$ is. Namely they could not show the quantitative value of $|\mathbf{T A}|$ or $|\mathbf{T A} / / \mathbf{T F}|$ for which their assumption is valid. If TA is almost perpendicular to $\mathbf{M F}$ (or TF) (perpendicular effect), the relative error (TIA-PTA)/PTA $(T I A)=\varepsilon_{\mathrm{T}} / P T A(T I A)$ is very large which affects significantly the analysis results. Using the actual observed TA, Figure F-1 shows the relation between relative error and $\beta$ which includes the perpendicular effects and provides the direct evaluation of the relative error for magnetization analysis as mentioned in the previous discussion. What we point out in our paper is that the assumption believed for long time so far that $T I A=P T A$ for $|\mathbf{T F}|>>|\mathbf{T A}|$ is not a good assumption because $\varepsilon_{\mathrm{T}}(=T I A-P T A)$ or $\varepsilon_{\mathrm{T}} / P T A(T I A)$ is the important parameter to evaluate analysis error. To get $\varepsilon_{\mathrm{T}}$, we need PTA, namely three component magnetic anomalies.

\section{Discussion - 5}

The questioner pointed out ' the amplitudes of such fluctuation to be much larger than $100 \mathrm{nT} \mathrm{p}-\mathrm{p}$, and that this is the noise level (probably coming from attitude data) of their three-component measurement in Aogashima. Such fluctuations could not be reflected in the 3D inversion results (figures 9-11), and the misfit in the inversion process (figure 8) remained over $200 \mathrm{nT}$ when expressed as a standard deviation, which is probably consistent with the values $2.5-3.5$ for the 'goodness-of-fit ratio'.

We understand now that the cause for the fluctuation was the instability of the RLG (Ring Laser Gyroscope) occurred when the helicopter suffered the rapid change of yaw, roll and pitch angles. We are preparing the next paper in which the fluctuations will be corrected better.

\section{Discussion - 6}

The questioner pointed out 'If the vector magnetic anomaly observation can be performed practically only under the error level more than $100 \mathrm{nT}$, and/or if the reproducibility by $3 \mathrm{D}$ inversion analysis of vector magnetic anomaly is limited to the misfit of $30 \%$, the peak difference ( $14 \mathrm{nT}$ in the example above) between $P T A$ and TIA will be of little importance. In my opinion, the development of the three-component magnetic survey system is actually one of the most important subjects to get over, but the high-resolution ability of total intensity measurements (owing to the developments of proton precession and optical pumping magnetometers) is still powerful paradigm even though the TIA does not give harmonic component in a strict sense.'

As mentioned in the text, the accuracy of the magnetometer sensor and positioning were $0.1 \mathrm{nT}$ and a few meters respectively. Due to the instability of the RLG, the accuracy of measurement as a whole, was regarded as less than 10nT. At present, we use NGSS positioning system with the accuracy less than a few $\mathrm{cm}$, and we now know the correction method for RLG instability, then the measurement accuracy as a whole will be less than a few nT.

We want to note that the accuracy of attitude and positioning of the magnetometer sensor is independent of the type of magnetometer. Even the optical pumping magnetometers with $0.01 \mathrm{nT}$ resolution must suffer the instability of RLG and the positioning error, additionally $\boldsymbol{\varepsilon}_{\mathrm{T}}$.

We emphasise again that any TIA measured even by a high accurate magnetometer could not make up the physical properties of TA. TA is a vector while $T I A$ is a scalar.

\section{Discussion - 7}

As the questioner pointed out, the legends in figure 5 were not correct. We appreciate his indication. We would like to take this opportunity to modify this legend of figure 5. Please see Figure F-2, the revised figure 5.

\section{Discussion - 8}

The questioner saw a strange saw-tooth feature in the observed data and calculated anomalies in figure 9. In this study, we obtained different altitude data at the same coordinates, therefore, the different values of the black and red points were plotted at the same distance (northward distance) from the origin.

In other words, our analysis was carried out to fit theoretical value to the observed data obtained at different altitudes. So, a strange saw-tooth feature appeared in the Figure (figure 9).

\section{Discussion - 9}

Questioner pointed out 'As is well known, the magnetic anomaly distribution cannot give unique solution of its causative source distribution. Then the analysis of magnetic anomaly has to depend on any restriction on the source distribution, and the strategic concept to overcome the difficulty should be given.'

We of course agree with this point. Then we repeat again that $T I A$ is not suitable data for the physical analysis, because the analysis error cased by $T I A-P T A$ cannot be evaluated, when we know only total intensity magnetic anomalies, TIA. This is clearly shown by Figure F-1.

\section{References}

Bhattacharyya, B. K., 1964, Magnetic anomalies due to prism-shaped bodies with arbitrary polarization: Geophysics, 29, 517-531. doi:10.1190/ 1.1439386

Blakely, R. J., 1995, Potential theory in gravity and magnetic applications: Cambridge University Press, 441p

Isezaki, N., and Matsuo, J., 2009, Magnetization structure of Aogashima Island using vector magnetic anomalies obtained by a helicopter-borne magnetometer: Exploration Geophysics, 40, 17-26; Butsuri-Tansa, 62, 17-26; Mulli-Tamsa, 12, 17-26. doi:10.1071/EG08112

Society of Exploration Geophysicists of Japan, 1999, Butsuri-Tansa handbook - Methodology: SEGJ, 944p (in Japanese)

Talwani, M., 1965, Computation with the help of a digital computer of magnetic anomalies caused by bodies of arbitrary shape: Geophysics, 30, 797-817. doi:10.1190/1.1439654

Manuscript received 24 August 2009; accepted 5 September 2009. 


\section{Appendix}
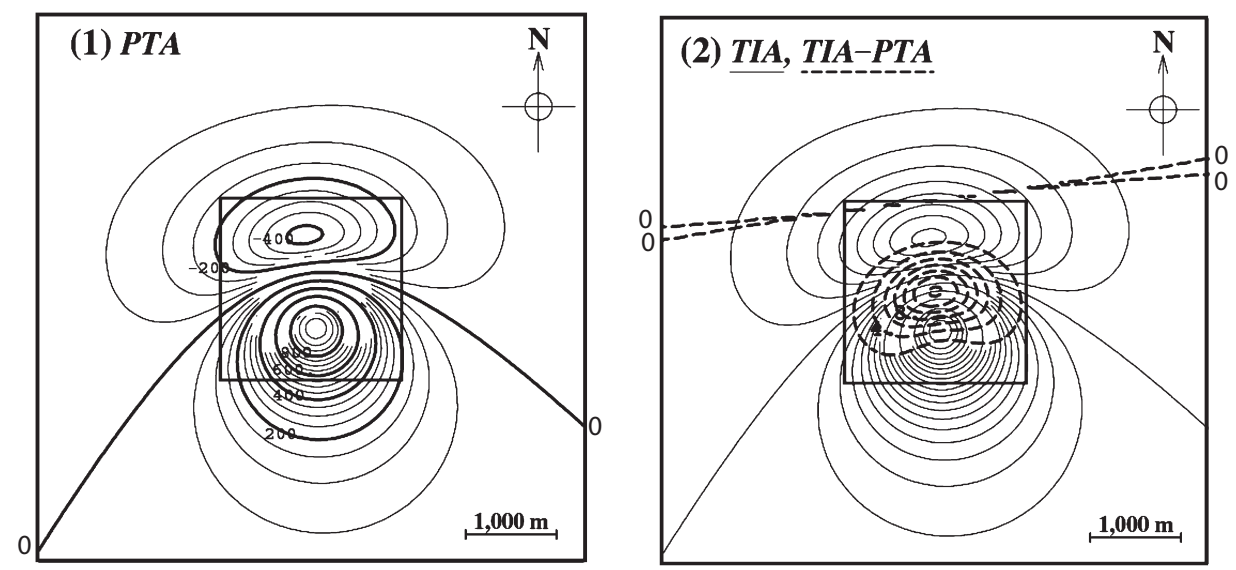

Fig. D-1. We suppose the model should be located at the red circle based on the question 2 . 